\title{
A percepção do enfermeiro da atenção primária acerca dos cuidados com os pés de indivíduos com Diabetes Mellitus
}

The perception of primary care nurses about the foot care of individuals with Diabetes Mellitus

La percepción de las enfermeras de atención primaria sobre el cuidado de los pies de las personas con Diabetes Mellitus

Recebido: 24/04/2021 | Revisado: 01/05/2021 | Aceito: 09/05/2021 | Publicado: 26/05/2021

Mariana Lopes Teixeira

ORCID: https://orcid.org/0000-0002-1382-3271

Universidade Estácio de Sá, Brasil

E-mail: marianalopestx@gmail.com

Larissa Lessa dos Santos

ORCID: https://orcid.org/0000-0002-8947-6691

Universidade Estácio de Sá, Brasil

E-mail: larissalessaa@hotmail.com

Angélica Cristina Castro Soares

ORCID: https://orcid.org/0000-0002-5202-6996

Universidade Castelo Branco, Brasil

E-mail: angelica.enf@outlook.com

Bruna Lira da Silva

ORCID: https://orcid.org/0000-0003-2668-620X Universidade do Grande Rio UNIGRANRIO, Brasil

E-mail: bruna.lira.56@gmail.com

Débora de Alencar Silva

ORCID: https://orcid.org/0000-0002-4379-0079 Universidade Estácio de Sá, Brasil

E-mail: deboraalencard@ hotmail.com

Eliane de Lira Goulart Caminha

ORCID: https://orcid.org/0000-0002-9061-2494 Universidade Veiga de Almeida, Brasil Universidade Federal do Estado do Rio de Janeiro, Brasil Albert Einstein, Brasil

E-mail: elianegoulart1984@gmail.com

Fábio Angelo de Medeiros

ORCID: https://orcid.org/0000-0002-2077-1457

Universidade Estácio de Sá, Brasil

E-mail: fabio.angelo36@gmail.com

Larissa Machado Campana Portela

ORCID: https://orcid.org/0000-0003-3248-2924

Universidade Veiga de Almeida, Brasil

E-mail: larissaenfuva@hotmail.com

Letícia Clementino dos Santos

ORCID: https://orcid.org/0000-0002-9316-9029

Universidade Estácio de Sá, Brasil

E-mail: leticiaenf2@outlook.com

Janaina Kelly da Silva de Souza de Araújo ORCID: https://orcid.org/0000-0001-7047-5712

Universidade Estácio de Sá, Brasil E-mail: jksilva756@gmail.com

Jéssica Pimentel Gomes da Costa Muchuli ORCID: https://orcid.org/0000-0002-6182-579X Universidade Iguaçu, Brasil E-mail: jp_jessik@hotmail.com

Marcus Vinícius Braga Esteves ORCID: https://orcid.org/0000-0001-5450-1693 Universidade Estácio de Sá, Brasil

E-mail:estevesbmarcus@outlook.com

Matheus Augusto Da Silva Belidio Louzada ORCID: https://orcid.org/0000-0001-9411-230X Escola de Enfermagem Aurora de Afonso Costa, Brasil E-mail: matheus.belidiolouzada@gmail.com 
Paloma de Oliveira Santos

ORCID: https://orcid.org/0000-0003-0679-3577 Universidade do Grande Rio UNIGRANRIO, Brasil E-mail: paloma.25enf@gmail.com

Priscilla Duarte Soares Correa

ORCID: https://orcid.org/0000-0002-9227-1259 Universidade Federal do Estado do Rio de Janeiro, Brasil Fundação Oswaldo Cruz, Brasil E-mail: priscillakdsoares@yahoo.com.br

Raiane Monteiro Rodrigues da Silva ORCID: https://orcid.org/0000-0002-1717-7254 Universidade Iguaçu, Brasil

E-mail: raiimonteiroo@gmail.com

Rodrigo de Albuquerque Lins

ORCID: https://orcid.org/0000-0003-2070-792X

Universidade Iguaçu, Brasil E-mail: rlins.rl@gmail.com

Vilma Regina Ferreira Rodrigues ORCID: https://orcid.org/0000-0002-8976-5477 Universidade do Estado do Pará, Brasi E-mail: enf.vilmarodrigues@gmail.com

Vítor Diego de Pontes Simões

ORCID: https://orcid.org/0000-0002-9317-4549

Centro Universitário Augusto Motta, Brasil E-mail: simoes872004@yahoo.com.br Vitória da Silva Araujo ORCID: https://orcid.org/0000-0003-2960-1347 Universidade Iguaçu, Brasil E-mail: vitoriasilva178@gmail.com

\begin{abstract}
Resumo:
Introdução: A Diabetes Mellitus (DM) estabelece um importante obstáculo para a saúde pública mantendo-se relacionado à qualificação das Unidades Básicas de Saúde (UBS). Por ser uma doença crônica, a DM pode acarretar danos sem possibilidades de serem revertidos e que refletem de maneira negativa na condição de vida dos indivíduos acometidos por esta patologia. Como decorrência do cuidado inadequado, podem surgir prejuízos nas extremidades do portador dessa patologia. É especialmente nas unidades básicas onde os funcionários da área da saúde precisam elaborar técnicas que viabilizem planos de cuidados que auxiliem na contribuição da assistência adequada e prevenção de agravos. Objetivo: Identificar através do estudo as dificuldades enfrentadas pelos profissionais e descrever quais as possíveis soluções para facilitar o cuidado para com a pessoa acometida por DM na atenção primária. Metodologia:Trata-se de um estudo de abordagem qualitativa, do tipo revisão integrativa no qual consta no procedimento de seis etapas que são a identificação do tema e seleção da hipótese, estabelecimento da estratégia de pesquisa, definição e coleta de dados, análise dos dados coletados, interpretação e apresentação dos resultados, onde será abordado o cuidado com o pé diabético na atenção básica. A estratégia de identificação e seleção dos artigos originou-se através da busca da Biblioteca Virtual de Saúde (BVS), com acesso as seguintes bases de dados eletrônicas como a Literatura Latino-Americana e do Caribe em Ciências da Saúde (LILACS), Medical Literature Analysis and Retrieval System Online (MEDLINE), Banco de Dados em Enfermagem (BDENF) e Scientific Electronic Library Online - Biblioteca Científica Eletrônica em Linha (SCIELO). Foram, também, realizadas buscas manuais nas listas de referências dos artigos selecionados no mês de março de 2021. Foram utilizados os seguintes descritores em ciências da saúde (DeCS): Diabetes mellitus; Pé diabético; Atenção primária e uso do boleador "and". Foram adotados os seguintes critérios de inclusão para seleção dos artigos: materiais completos, em português e inglês, no formato de artigo e com recorte temporal de 2010 a 2020. Os critérios de exclusão foram os artigos duplicados, incompletos e que não atendiam a temática do estudo. Resultados: Após pesquisa minuciosa foram encontradas duas categorias: 1- A importância da prevenção das lesões no pé diabético para evitar danos irreversíveis à saúde e 2- $\mathrm{O}$ entendimento do público diabético quanto aos cuidados com os pés. Considerações Finais: Ao final da pesquisa foi possível concluir a importância da assistência fornecida pelo enfermeiro ao paciente diabético na Atenção Básica de Saúde. Sua função vai muito além de avaliar o estado geral do cliente, mas também há um papel de educador.
\end{abstract}

Palavras-chave: Diabetes mellitus; Pé diabético; Atenção primária.

\begin{abstract}
:
Introduction: Diabetes Mellitus (DM) establishes an important obstacle for public health, remaining related to the qualification of Basic Health Units (UBS). As it is a chronic disease, DM can cause damage without the possibility of being reversed and which reflect negatively on the life conditions of individuals affected by this pathology. As a result of inadequate care, damage may occur in the extremities of the carrier of this pathology. It is especially in the basic units where health care workers need to develop techniques that make care plans feasible to assist in the contribution
\end{abstract}


of adequate care and disease prevention. Objective: To identify through the study the difficulties faced by professionals and describe what are the possible solutions to facilitate care for the person affected by DM in primary care. Methodology: This is a qualitative approach study, of the integrative review type, which is part of the six-step procedure, which is the identification of the theme and selection of the hypothesis, establishment of the research strategy, definition and data collection, data analysis collected, interpretation and presentation of results, which will address the care of diabetic foot in primary care. The strategy of identification and selection of articles originated through the search for the Virtual Health Library (VHL), with access to the following electronic databases such as Latin American and Caribbean Literature in Health Sciences (LILACS), Medical Literature Analysis and Retrieval System Online (MEDLINE), Nursing Database (BDENF) and Scientific Electronic Library Online - Electronic Scientific Library Online (SCIELO). Manual searches were also carried out on the reference lists of selected articles in March 2021. The following descriptors in health sciences (DeCS) were used: Diabetes mellitus; Diabetic foot; Primary care and use of the "and" boleador. The following inclusion criteria were adopted for the selection of articles: complete materials, in Portuguese and English, in article format and with a time frame from 2010 to 2020. The exclusion criteria were duplicate, incomplete articles that did not meet the theme of the study. Results: After thorough research, two categories were found: 1- The importance of preventing injuries to the diabetic foot to avoid irreversible damage to health and 2- The understanding of the diabetic public regarding foot care. Final Considerations: At the end of the research, it was possible to conclude the importance of the assistance provided by nurses to diabetic patients in Primary Health Care. Their role goes far beyond assessing the general condition of the client, but there is also an educator role.

Keywords: Diabetes mellitus; Diabeticfoot; Primaryattention.

\section{Resumen:}

Introducción: La Diabetes Mellitus (DM) establece un importante obstáculo para la salud pública, quedando relacionado con la calificación de las Unidades Básicas de Salud (UBS). Al tratarse de una enfermedad crónica, la DM puede causar daños sin posibilidad de reversión y que repercuten negativamente en las condiciones de vida de los individuos afectados por esta patología. Como resultado de una atención inadecuada, pueden producirse daños en las extremidades del portador de esta patología. Es especialmente en las unidades básicas donde los trabajadores de la salud necesitan desarrollar técnicas que viabilicen los planes de atención para ayudar en la contribución de la atención adecuada y la prevención de enfermedades. Objetivo: Identificar a través del estudio las dificultades que enfrentan los profesionales y describir cuáles son las posibles soluciones para facilitar la atención a la persona afectada por DM en atención primaria. Metodología: Se trata de un estudio de enfoque cualitativo, del tipo revisión integradora, que forma parte del procedimiento de seis pasos, que consiste en la identificación del tema y selección de la hipótesis, establecimiento de la estrategia de investigación, definición y recolección de datos, análisis recogido, interpretación y presentación de resultados, que abordarán la atención del pie diabético en atención primaria. La estrategia de identificación y selección de artículos se originó a través de la búsqueda de la Biblioteca Virtual en Salud (BVS), con acceso a las siguientes bases de datos electrónicas como Literatura Latinoamericana y del Caribe en Ciencias de la Salud (LILACS), Sistema de Análisis y Recuperación de Literatura Médica en Línea (MEDLINE), Base de datos de enfermería (BDENF) y Biblioteca electrónica científica en línea - Biblioteca científica electrónica en línea (SCIELO). También se realizaron búsquedas manuales en las listas de referencias de los artículos seleccionados en marzo de 2021. Se utilizaron los siguientes descriptores en ciencias de la salud (DeCS): Diabetes mellitus; Pie diabético; Atención primaria y uso del "y" boleador. Se adoptaron los siguientes criterios de inclusión para la selección de artículos: materiales completos, en portugués e inglés, en formato de artículo y con un marco temporal de 2010 a 2020. Los criterios de exclusión fueron artículos duplicados, incompletos que no cumplían con la temática del estudio. Resultados: Después de una investigación exhaustiva, se encontraron dos categorías: 1- La importancia de prevenir las lesiones en el pie diabético para evitar daños irreversibles a la salud y 2- La comprensión del público diabético sobre el cuidado del pie. Consideraciones finales: Al finalizar la investigación se pudo concluir la importancia de la asistencia que brindan las enfermeras a los pacientes diabéticos en Atención Primaria de Salud. Su rol va mucho más allá de evaluar el estado general del cliente, pero también hay un educador papel.

Palabras clave: Diabetes mellitus; Pie diabético; Atencion primaria.

\section{Introdução}

A Diabetes Mellitus (DM) estabelece um importante obstáculo para a saúde pública mantendo-se relacionado à qualificação das Unidades Básicas de Saúde (UBS). Aproximadamente 415 milhões de pessoas possuem DM com predomínio de $8,8 \%$ no mundo. O Brasil encontra-se na quarta posição entre os países com mais indivíduos com DM mundialmente, e calcula-se que nos dias atuais 14,3 milhões de brasileiros são diabéticos. Lira et al. (2020). 
Por ser uma doença crônica, a DM pode acarretar danos sem possibilidades de serem revertidos e que refletem de maneira negativa na condição de vida dos indivíduos acometidos por esta patologia. Além do mais, lesões nos pés em consequência do pé diabético são grandes razões de internações hospitalares e remoção de extremidade. Silva et al. (2020).

Os pés de um paciente diabético geralmente apresentam lesões, contaminação e/ou perda de tecidos muito fundos, habitualmente relacionados a distúrbios neurológicos e à patologia de vasos periféricos. Como decorrência do cuidado inadequado, podem surgir prejuízos nas extremidades do portador de DM. É necessária avaliação periódica dos membros para o planejamento e recurso terapêutico das irregularidades descobertas, assim, prevenindo agravos. Lira et al. (2020).

O exame do risco de lesão fundamenta-se em apurar motivos que contribuem para a evolução do pé diabético, através de testes clínicos e laboratoriais. Por meio dessa técnica, o profissional enfermeiro, na assistência da coletividade com DM, apresenta as intervenções, as orientações necessárias, imprescindíveis no atendimento de Enfermagem para certificar a plenitude do atendimento. Lira et al. (2020).

É especialmente na atenção primária onde os funcionários da área da saúde precisam elaborar técnicas que viabilizem planos de cuidados que auxiliem na contribuição da assistência adequada e prevenção de agravos. A aplicação de manuais é um recurso que pode ser empregado pelo enfermeiro para a referência e identificação de casos específicos. Nascimento et al. (2019).

A prevenção é o primeiro passo para combater as úlceras diabéticas. Os profissionais devem também orientar aos pacientes sobre a necessidade de observar os membros e avaliar presença de edema, eritema, coloração dos pés, perfurações, desidratação, calosidades, entre outros sinais. Cuba et al. (2013)

A partir do exposto, faz-se necessário o conhecimento do enfermeiro da Atenção Básica sobre os cuidados voltados aos pés dos usuários acometidos pela Diabetes Mellitus. É de extrema importância que o profissional possua habilidade e conhecimento para realizar o atendimento sendo capaz de orientar esse paciente de maneira adequada.

Nesta perspectiva, a questão que subsidiou a pesquisa foi: Qual as dificuldades enfrentadas pelo profissional enfermeiro da atenção primária no cuidado com o pé diabético? Assim, o presente estudo apresenta como objeto "as dificuldades do cuidado com o pé diabético, enfrentadas pelo enfermeiro da atenção primária".

O objetivo da pesquisa é identificar através do estudo as dificuldades enfrentadas pelos profissionais e descrever quais as possíveis soluções para facilitar o cuidado para com a pessoa acometida por DM na atenção primária.

O estudo se justifica a partir do elevado número de casos de pacientes portadores de DM nas unidades básicas que apresentam ulcerações nos pés decorrentes da doença. A partir do exposto fica clara a importância da elaboração de uma revisão de literatura destacando a temática.

A relevância para os acadêmicos de enfermagem se faz com a intenção de informar e buscar uma reflexão sobre a temática, uma vez que será de grande importância para a assistência realizada por eles no futuro; social por levar à sociedade o conhecimento científico ao qual, de costume, ela não teria acesso, e para fins de pesquisa, já que outros poderão então fazer uso dos dados encontrados e aqui reunidos e analisados.

\section{Metodologia}

Bardin (2016), define que a pesquisa qualitativa é a que se fundamenta principalmente em análises qualitativas, caracterizando-se, em princípio, pela não utilização de instrumental estatístico na análise dos dados.

Não é apenas a "pesquisa não quantitativa", tendo desenvolvido sua própria identidade. Assim, visa entender, descrever e explicar os fenômenos sociais de modos diferentes, através da análise de experiências individuais e grupais, exame de interações e comunicações que estejam se desenvolvendo, assim como da investigação de documentos (textos, imagens, filmes ou músicas) ou traços semelhantes de experiências e integrações (Flick, 2009). 
Também possui caráter descritivo, cujo foco não consiste na abordagem, mas sim no processo e seu significado, ou seja, o principal objetivo é a interpretação do fenômeno objeto de estudo (Silva; Menezes, 2005).

A revisão integrativa visa fazer uma interseção de estudos da mesma linha de conhecimento, com o objetivo de analisar e sintetizar os mesmos, para que se obtenha uma informação mais abrangente de um fenômeno específico (Cooper,1984).

Segundo Ganong (1987) a revisão integrativa da literatura propõe o estabelecimento de critérios bem definidos sobre a coleta de dados, análise e apresentação dos resultados, desde o início do estudo, a partir de um protocolo de pesquisa previamente elaborado e validado.

Para a construção do artigo abordou-se a revisão integrativa da literatura, que consiste na elaboração de análise abrangente de artigos, estabelecendo critérios de investigação técnico-científico para coletar dados, analisar e apresentar resultados. As informações obtidas podem sinalizar achados que requerem mais atenção no meio científico, visando melhorias na prática profissional (Ganong, 1987).

A revisão integrativa necessita ser guiada por etapas. De acordo com Mendes, Silveira e Galvão (2008) essa revisão deverá conter seis etapas: 1) Estabelecimento de hipótese ou questão de pesquisa; 2) Amostragem ou busca na literatura; 3) Categorização dos estudos; 4) Avaliação dos estudos incluídos na revisão; 5) Interpretação dos resultados; 6) Síntese do conhecimento ou apresentação da revisão.

A estratégia de identificação e seleção dos artigos originou-se através da busca da Biblioteca Virtual de Saúde (BVS), no mês de março de 2021. Iniciou-se a busca de materiais que contivessem em seu resumo os descritores em ciências da saúde (DeCS) Diabetes Mellitus; Pé diabético; Atenção Primária. Foram adotados os seguintes critérios para seleção dos artigos: materiais completos, em língua portuguesa, no formato de artigo e com recorte temporal de cinco anos (2010-2020). Os critérios de exclusão foram os estudos que não atendessem aos critérios citados anteriormente e duplicados.

Emergiram 5 artigos com os critérios citados, os quais procederam-se à leitura minuciosa para a construção do conteúdo, destacando aqueles que responderam ao objetivo proposto pelo estudo, com intuito de organizar os dados.

Os artigos foram tabelados por título, autores, base de dados, ano de publicação, objetivo, método de pesquisa e conclusão. A partir de então iniciou a análise bibliomética desses que foram agrupados por similaridade sob forma de categorias.

\section{Resultados e Discussão:}

Os resultados apresentados no estudo foram descritos no Quadro 1. 
Quadro 1: Características dos artigos analisados no período de 2010 a 2020.

\begin{tabular}{|c|c|c|c|c|c|c|}
\hline Título & Autoria & $\begin{array}{c}\text { Base de } \\
\text { dados }\end{array}$ & Ano & Objetivo & Métodos & $\begin{array}{c}\text { Considerações } \\
\text { Finais }\end{array}$ \\
\hline $\begin{array}{l}\text { Conhecimento do } \\
\text { enfermeiro acerca } \\
\text { dos cuidados com } \\
\text { o pé diabético }\end{array}$ & $\begin{array}{c}\text { Luana } \\
\text { Savana; } \\
\text { Catiane } \\
\text { Raquel; } \\
\text { Roberto } \\
\text { Wagner; } \\
\text { Ana Larissa; } \\
\text { Luisa } \\
\text { Helena; } \\
\text { Ana Roberta. }\end{array}$ & BDENF & 2019 & $\begin{array}{c}\text { Compreender o } \\
\text { conhecimento do } \\
\text { enfermeiro sobre o } \\
\text { cuidado com os pés de } \\
\text { pacientes diabéticos. }\end{array}$ & $\begin{array}{c}\text { Estudo } \\
\text { quantitativo e } \\
\text { descritivo. }\end{array}$ & $\begin{array}{c}\text { Identificou-se } \\
\text { conhecimento } \\
\text { insatisfatório, } \\
\text { destacando a } \\
\text { necessidade de } \\
\text { programas de } \\
\text { atualização das } \\
\text { práticas educativas } \\
\text { quanto à avaliação } \\
\text { dos pés.. }\end{array}$ \\
\hline $\begin{array}{c}\text { Construção e } \\
\text { validação de um } \\
\text { manual de } \\
\text { detecção do pé } \\
\text { diabético para } \\
\text { atenção primária }\end{array}$ & $\begin{array}{c}\text { José } \\
\text { William; } \\
\text { Edjôse } \\
\text { Ciríaco; } \\
\text { Manoel } \\
\text { Luiz; } \\
\text { Suzane } \\
\text { Brust. }\end{array}$ & $\begin{array}{c}\text { Revista } \\
\text { Oficial do } \\
\text { COFEN }\end{array}$ & 2019 & $\begin{array}{c}\text { Descrever o processo de } \\
\text { construção e validação } \\
\text { de um manual do pé } \\
\text { diabético para } \\
\text { profissionais da saúde } \\
\text { da Atenção Primária. }\end{array}$ & $\begin{array}{c}\text { Pesquisa } \\
\text { metodológica. }\end{array}$ & $\begin{array}{l}\text { Manual apresentou } \\
\text { fidedignidade e } \\
\text { confiabilidade para } \\
\text { a observação da } \\
\text { qualidade da } \\
\text { assistência. }\end{array}$ \\
\hline $\begin{array}{l}\text { Grau de risco do } \\
\text { pé diabético na } \\
\text { atenção primária à } \\
\text { saúde }\end{array}$ & $\begin{array}{l}\text { Patrícia } \\
\text { Simon; } \\
\text { Cassandra } \\
\text { Severo; } \\
\text { Ludmila } \\
\text { Mourão; } \\
\text { Thiago Luis. }\end{array}$ & REUFSM & 2020 & $\begin{array}{l}\text { Investigar o grau de } \\
\text { risco de pé diabético e } \\
\text { fatores associados em } \\
\text { indivíduos com DM. }\end{array}$ & $\begin{array}{c}\text { Estudo } \\
\text { transversal. }\end{array}$ & $\begin{array}{c}\text { A maioria dos } \\
\text { pacientes não } \\
\text { possuem alto risco, } \\
\text { porém o enfoque } \\
\text { deve ser no } \\
\text { autocuidado para } \\
\text { prevenção de } \\
\text { agravos. }\end{array}$ \\
\hline $\begin{array}{l}\text { Conhecimento do } \\
\text { enfermeiro sobre a } \\
\text { prevenção do pé } \\
\text { diabético: Revisão } \\
\text { Integrativa da } \\
\text { Literatura }\end{array}$ & $\begin{array}{c}\text { Luana } \\
\text { Savana; } \\
\text { Malvina } \\
\text { Thaís; } \\
\text { Márcio } \\
\text { Dênis; } \\
\text { Ana Roberta. }\end{array}$ & $\begin{array}{c}\text { Revista } \\
\text { Brasileira } \\
\text { em } \\
\text { Promoção } \\
\text { da Saúde }\end{array}$ & 2017 & $\begin{array}{c}\text { Sistematizar o } \\
\text { conhecimento do } \\
\text { enfermeiro a respeito do } \\
\text { pé diabético. }\end{array}$ & $\begin{array}{l}\text { Revisão } \\
\text { integrativa de } \\
\text { literatura. }\end{array}$ & $\begin{array}{l}\text { Conclui-se que as } \\
\text { orientações } \\
\text { determinadas pelas } \\
\text { diretrizes para } \\
\text { prevenir o pé } \\
\text { diabético torna-se } \\
\text { insuficiente. }\end{array}$ \\
\hline $\begin{array}{l}\text { Condutas dos } \\
\text { enfermeiros da } \\
\text { atenção primária } \\
\text { no cuidado a } \\
\text { pessoas com pé } \\
\text { diabético }\end{array}$ & $\begin{array}{l}\text { Caroline } \\
\text { Porcelis; } \\
\text { Daniella } \\
\text { Karine; } \\
\text { Dhayana } \\
\text { Loyze; } \\
\text { Soraia } \\
\text { Dornelles; } \\
\text { Mara } \\
\text { Ambrosina; } \\
\text { Soraia } \\
\text { Geraldo. }\end{array}$ & REUOL & 2017 & $\begin{array}{c}\text { Conhecer as ações do } \\
\text { enfermeiro da atenção } \\
\text { primária no cuidado das } \\
\text { pessoas com diabetes } \\
\text { mellitus. }\end{array}$ & $\begin{array}{c}\text { Pesquisa } \\
\text { qualitativa. }\end{array}$ & $\begin{array}{l}\text { Foi compreendido } \\
\text { que a conduta dos } \\
\text { enfermeiros quanto } \\
\text { a essa prática com } \\
\text { os cuidados dos } \\
\text { pés na atenção } \\
\text { primária é ineficaz. }\end{array}$ \\
\hline
\end{tabular}

Fonte: Autores. 
Após análise dos artigos selecionados neste estudo foram construídas as seguintes categorias:

Categoria I - A importância da prevenção das lesões no pé diabético para evitar danos irreversíveis à saúde.

Levando em consideração que o pé diabético se configura como um dos distúrbios mais importantes do portador de Diabetes Mellitus (DM), sujeitos de grande intermédio nas Unidades Básicas de Saúde, a construção de um instrumento de precaução e percepção precoce é inclusive, uma forma de padronizar a assistência e certificar os planos de prevenção, por meio de uma equipe multidisciplinar. Nascimento et al. (2019).

Através do reconhecimento da fisiologia, patologia e etiologia do pé diabético, pacientes que apresentam maior ameaça de agravamento são apontados de forma precoce, impossibilitando o surgimento de lesões ulcerativas e amputações. De acordo com pesquisas, 50\% das retiradas de membros podem ser impedidas por meio de orientação e assistência interdisciplinar fundamentada em comprovações científicas. Nascimento et al. (2019).

O conhecimento de pacientes em risco, através do rastreamento metódico do pé diabético, é capaz de levar à redução exorbitante dos casos de amputações dos membros inferiores, alcançando visíveis vantagens na saúde e do bem estar. O costume de andar sem calçados é extremamente prejudicial, pesquisadores pedem para que as pessoas fiquem alerta sobre esse comportamento, pois o mesmo facilita a aparição ou complicação de lesões nos pés. Silva et al. (2016).

Podemos perceber que a compreensão sobre os cuidados que o paciente deve realizar e dominar são muito escassos entre a equipe de saúde, visto que as instruções objetivam cortar as unhas corretamente, utilizar sapato adequado e observar mudanças nos pés, à medida que as precauções devem ser integrais, porque a diabetes mellitus é uma patologia de caráter sistêmico, sendo necessário trabalhá-la holisticamente. Sousa et al. (2017).

A orientação para o cuidado próprio das pessoas possibilita que as mesmas tenham diversos benefícios e um cuidado digno. É importante inspecionar os pés e examiná-los como uma atividade terapêutica, da mesma maneira que utilizam as medicações para controlar a DM. Silva et al. (2016).

Para a execução da examinação dos pés, o usuário deve tirar os sapatos e o avaliador terá que fazer uma análise minuciosa dos pés em um espaço com iluminação adequada. $\mathrm{O}$ avaliador deve também inspecionar se os calçados são corretos e trazem conforto aos pés. Sousa et al. (2017).

É indispensável observar o aspecto da pele, englobando o julgamento da higiene, unhas, existência de infecções e/ou não de lesões ou regiões de rubor. O avaliador analisa também se há deformação no qual as habituais, locais de pressão plantar elevadas, rachaduras na pele ou dedo no formato de garra. Sousa et al. (2017).

É válido lembrar que existe uma análise vascular, em que os pulsos tibial e posterior são tateados e descritos como presentes ou ausentes. Já a análise neurológica tem como propósito julgar a ausência de percepção protetora através de testes. Sousa et al. (2017).

Recomenda-se que todo portador de DM efetue, no mínimo anualmente, a avaliação dos pés. O profissional deve contar com determinadas circunstâncias que representam condições de risco para a evolução do pé diabético, como amputações anteriores, história de lesões nos pés, neuropatias, deformidades, doenças vasculares, descontrole glicêmico, tabagismo e outros. Sousa et al. (2017).

Assim, é dada ênfase no auto-cuidado, reduzindo ou excluindo qualquer dano ou perigo para o aparecimento de feridas, trazendo melhor qualidade de vida. É necessário criação de vínculo e troca entre o profissional e o paciente, onde o enfermeiro procure sensibilizar o usuário, incentivando algumas mudanças no estilo de vida, fazendo-o refletir de maneira não abstrata, deixando-o informado sobre seu caso, tornando-o protagonista do seu tratamento. Silva et al. (2016).

É importante enfatizar que o projeto de Educação Permanente em Saúde contribui para beneficiar e ajudar na capacitação dos enfermeiros, padronizando e sistematizando a assistência ao paciente com diabetes integralmente. Sousa et al. (2017). 
O estudo demonstra que, mesmo não havendo uma assistência padronizada, os usuários com pé diabético nas UBS são atendidos de maneira racional, através de uma comunicação interdisciplinar que beneficia e evita possível agravamento.

Categoria II - O entendimento do público diabético quanto aos cuidados com os pés.

O distúrbio metabólico da Diabeter Mellitus gera comprometimento nos níveis de glicose e reservas de energia, podendo gerar inúmeras complicações em órgãos vitais, gerando lesões incapacitantes. A mais comum é a complicação nos pés, conhecida como pé diabético. Essa síndrome tem histórico neuropático e é ocasionada pela hiperglicemia sustentada, podendo gerar deformidades, ulcerações, infecções e traumatismos. Vargas et al. (2017).

É fundamental que o paciente conheça a importância de realizar o acompanhamento ambulatorial de forma anual e dos cuidados que devem ser realizados para que o mesmo previna tais lesões indesejáveis que podem complicar e gerar inclusive a perda do membro inferior. Vargas et al. (2017).

Defronta-se, pelo indivíduo com pé diabético, no dia a dia, forte declínio socioeconômico, no que diz respeito ao emocional e também questões biológicas, que compreendem despesas, intervenções e dificuldades físicas, fatores que atrapalham o seu auto-cuidado e a qualidade de vida do paciente. Arruda et al. (2019).

Alguns pacientes possuem conhecimento ineficaz sobre os cuidados necessários que devem ser realizados para preservar a saúde dos pés, por essa razão, os profissionais de saúde devem orientá-los durante as consultas e explicar as práticas simples que os mesmos podem fazer rotineiramente e que são eficazes para a prevenção de lesões. Arruda et al. (2019).

De acordo com o Manual do Pé Diabético (2016), a primeira forma avaliativa dos pés que os pacientes precisam aprender é a inspeção. É necessário olhar os pés diariamente, avaliando a coloração, se há deformidade, calos, edemas; algum corte ou ferida.Outra conduta necessária é a percepção da temperatura no membro, é importante observar se está frio, morno ou quente.

Os profissionais de saúde devem orientar sobre o uso de meias sem costura devido a pressão que é exercida em eixos pontuais do membro, escolha de sapatos confortáveis e adequados, corte correto das unhas, higienização dos pés, a importância de secar entre os dedos dos pés após tomar banho, assim como ensinar técnicas de limpeza e condutas que devem ser realizadas quando há ferimento presente. Manual do Pé Diabético (2016).

\section{Considerações Finais:}

Ao final da pesquisa foi possível concluir a importância da assistência fornecida pelo enfermeiro ao paciente diabético na Atenção Básica de Saúde. Sua função vai muito além de avaliar o estado geral do cliente, mas também há um papel de educador quando o mesmo fornece orientações básicas e não menos importantes para que o próprio portador da doença conheça sua patologia e seus riscos.

Quando o usuário é bem orientado quanto a medidas de prevenção para evitar as lesões nos pés, assim como os métodos de observância nos locais importantes, o risco para que ocorra uma lesão é reduzido expressivamente e até mesmo se a lesão já estiver instalada, a probabilidade de um bom prognóstico devido a detecção precoce é excelente.

Nota-se a importância do olhar do enfermeiro na Saúde Pública, fazendo a diferença em seu atendimento e na vida dos usuários, ensinando-lhes técnicas diárias simples que previnem e impedem a evolução de úlceras.

Aguarda-se que este estudo promova a análise reflexiva dessas práticas pelos futuros profissionais e conscientize os enfermeiros já atuantes na área que não realizam educação em saúde com seus pacientes, potencializando assim, a atividade educativa nos setores de saúde. 
Research, Society and Development, v. 10, n. 5, e14810615415, 2021

(CC BY 4.0) | ISSN 2525-3409 | DOI: http://dx.doi.org/10.33448/rsd-v10i6.15415

\section{Referências}

Arruda, L. S. N. S., Fernandes, C. R. S., Freitas, R. W. J. F., Machado, A.L. G., Lima, L. H. O., \& Silva, A. R. V. Conhecimento do enfermeiro acerca dos cuidados com o pé diabético. Revenferm UFPE online. 2019;13:e242175

Bardin, L. (2016). Análise de conteúdo. Almedina Brasil.

Cooper, H. M. Diretrizes científicas para conduzir revisões integrativas depesquisa. Review of Educational Research, 52(2), 291-302.

Cubas, M. R., Santos, O. D., Retzlaff, E. M., Telma, H. L., Andrade, I. P., Moser, A. D., \& Erzinger, A. R. (2013). Pé diabético: orientações e conhecimento sobre cuidados preventivos. Fiosioter. Mov., 647-55.

Flick, U. Desenho da pesquisa qualitativa. Artmed.

Ganong, L. H. (1987). Integrative reviews of nursing research. New Tork, 10(11), 1-11.

Lira, J. A. Ca. et al. Avaliação do risco de ulceração nos pés em pessoas com Diabetes Mellitus. na atenção primária.. Reme: Rev. Min. Enferm., 24 , e1327.

Mendes, K., Silveira, R. C., \& Galvão, C. M. (2008). Revisão integrativa: método de pesquisa para a incorporação de evidências na saúde e na enfermagem. Texto Contexto Enfermagem, 17(4): 758-64.

Ministério da Saúde. Manual do Pé Diabético. (2016).

Nascimento, J.W. A., et al Construção e validação de um manual do pé diabético para atenção primária. Enferm. Foco 2019 ; 10 (6): $85-9185$.

Santana da Silva, L. W., et al (2016). Cuidados de los pies de personas con diabetes mellitus: acciones protectoras vinculadas a la promoción de la salud. Enfermería: Cuidados Humanizados, 5(2), 12-18.

Silva, P. S., et al. Grau de risco do pé diabético na atenção primária à saúde. Rev. Enferm. UFSM. 10, e78, 1-16, 2020.

Silva, E. L., \&Menezes, E. M. Metodologia da pesquisa e elaboração de dissertação. (4a. ed.).

Sousa, L. S. N. et al. Conhecimento do enfermeiro sobre a prevenção do pé diabético: revisão integrativa da literatura. Revista Brasileira em promoção de saúde.

Vargas, C. P., Lima, D. K., Silva, D. L., Schoeller, S. D., Vragas, M. A., \& Lopes, S. G. (2017). Condutas dos enfermeiros da atenção primária no cuidado a pessoas com pé diabético. Revista de Enfermagem UFPE On-line. 